\title{
Using Frequency-Domain Methods to Identify XV-15 Aeroelastic Modes
}

C. W. Acree, Jr. and Mark B. Tischler (AASA-TH-100033) OSING PEECOENCY-DOHAIN
EETECDS TC IDENIIFY XV-15 AEECELASTIC MODES
(YASA) $25 \mathrm{P}$
CSCL OIC

November 1987

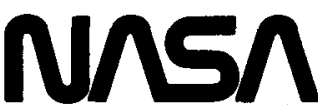

National Aeronautics and

Space Administration
N88-17646

unclas

0125854 


\section{Using Frequency-Domain Methods to Identify XV-15 Aeroelastic Modes}

C. W. Acree, Jr., Ames Research Center, Moffett Field, California

Mark B.Tischler, Aeroflightdynamics Directorate, U.S. Army Aviation Research and

Technology Activity, Ames Research Center, Moffett Field, California

November 1987

\section{N/SA}

National Aeronautics and

Space Administration 
USING FREQUENCY-DOMAIN METHODS TO

IDENTIFY XV-15 AEROELASTIC MODES

\author{
C. W. Acree, Jr. \\ Ames Research Center \\ Mark B. Tischler \\ Aeroflightdynamics Directorate \\ U.S. Army Aviation Research and Technology Activity \\ Ames Research Center
}

SUMMARY

The XV-15 Tilt-Rotor wing has six major aeroelastic modes that are close in frequency. To precisely excite individual modes during flight test, dual flaperon exciters with automatic frequency-sweep controls were installed. The resulting structural data were analyzed in the frequency domain (Fourier-transformed) with cross-spectral and transfer-function methods. Modal frequencies and damping were determined by performing curve fits to transfer-function magnitude and phase data and to cross-spectral magnitude data. Results are given for the XV-15 with its original metal rotor blades. Frequency and damping values are also compared with earlier predictions.

\title{
INTRODUCTION
}

Distinctive features of the XV-15 Tilt Rotor are the large wing-tip pylons which house the engines, transmissions and pivoting mechanisms for each rotor (fig. 1). The highest speeds are obtained in the cruise mode, making it the critical operating mode for aeroelastic stability. The concentrated masses at the wing tips keep the modal frequencies fairly low. Also, aeroelastic coupling between each rotor and pylon is destabilizing. Consequently, close attention must be paid to potential whirl-mode flutter during flight test. The problem is not unique to the $\mathrm{XV}-15$ research aircraft, but is fundamental to any tilt-rotor aircraft of similar configuration, such as the XV-3, for which extensive studies were done (refs. 1 and 2), and the upcoming V-22 Osprey. The impact of aeroelastic stability requirements on tilt-rotor design is discussed in reference 3 .

The planned flight tests of new composite rotors on the XV-15 (ref. 4) provided the incentive for a thorough reevaluation of XV-15 aeroelastics using the latest flight-test and modal identification techniques. The major wing modes were excited with flaperon frequency sweeps. Modal frequencies and damping were determined by performing curve fits to transfer-function magnitude and phase data and to crossspectral magnitude data. The analysis programs have been used successfully on other flight data, notably for XV-15 aircraft flight dynamics (ref. 5). 
ORIGINAL PAGE IS OE POOR QUALTTY
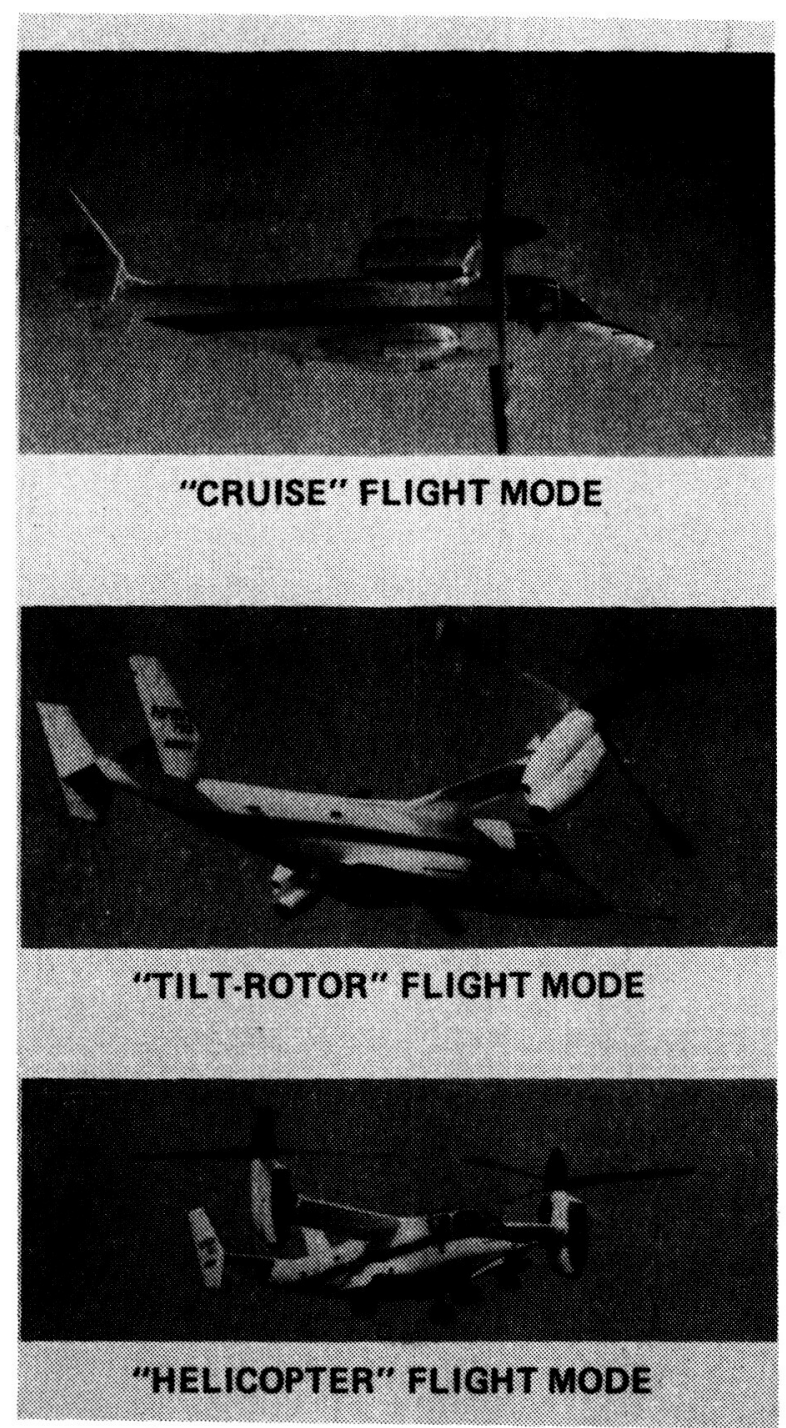

Figure 1.- XV-15 tilt-rotor research aircraft.

More complete discussions of the aeroelastic behavior of the XV-15, including previous flight-test data, are given in references 6 and 7 . All flight-test data discussed in the present report are for the original metal blades and new steel hubs. References 6 and 7 give data for the metal blades and original titanium hubs.

This report presents discussions of the XV-15 aeroelastic modes and the flighttest techniques used to excite them; the analytical procedures used to extract modal frequencies and damping from flight-test data; and plots of estimated frequency and damping versus airspeed, including comparisons with theoretical values.

\section{FLIGHT-TEST METHODS}

The six major wing/pylon aeroelastic modes are illustrated in figures 2 and 3 and are listed in figure 4, which shows the ranges of natural frequencies identified during the flight-test program. Note that some predictions were inaccurate, even 


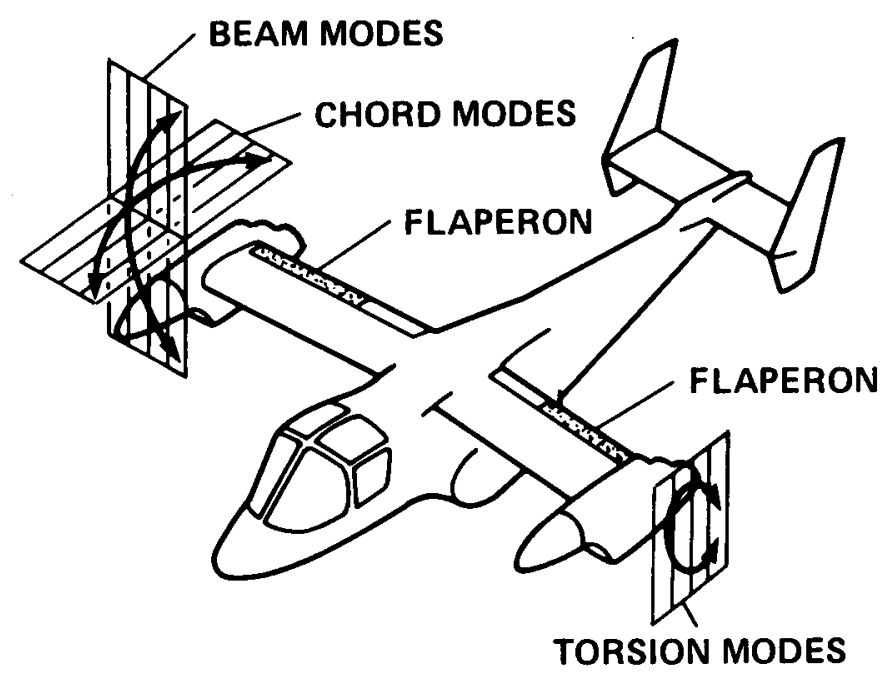

Figure 2.- XV-15 aircraft, showing flaperons and wing modes.

though they include refinements made after the aircraft was initially tested (ref. 6). The predictions and experimental data together indicate that at least one mode lies within the design rotor-speed range. Consequently, the rotor speed is restricted in cruise to $8.6 \mathrm{~Hz}(86 \%$ of $601 \mathrm{rpm})$ instead of the design minimum of $7.6 \mathrm{~Hz}$. Several modes are very close; two--the antisymmetric-chord and antisymmetric-torsion modes--actually overlap, which was not predicted. It is this close placement in frequency and restricted rotor-speed range that makes precise identification of individual modes both difficult and necessary.

In earlie' flight tests (refs. 6 and 7), frequency and damping were identified by using primarily the exponential-decay technique with a Prony analysis (ref. 8). The natural frequencies were precisely identified, but the damping values showed a great deal of scatter, especially where neighboring modes were grouped closely together. Limited data were obtained using turbulence excitation and analyzed with RANDOMDEC techniques (ref. 6), but again with considerable scatter. Frequency sweeps were also tried, but the original exciters were unable to adequately excite the modes (ref. 7).

Additional flight tests (ref. 5) used turbulence excitation, followed by frequency-domain analyses to identify frequencies and damping. The turbulence did not excite the modes strongly enough in most cases. However, the results of those tests indicated that the frequency-domain method was the most promising approach, provided that dual flaperon exciters were used to selectively excite the symmetric and antisymmetric modes, as recommended in reference 6 . Compared to the exponential-decay and RANDOMDEC methods, the frequency-domain method is less sensitive to noise and makes more efficient use of flight time; accordingly, it is the method of choice.

The earlier flight tests used a high-frequency, limited-authority servo actuator in series with the right-flaperon control linkage to excite the bending and 


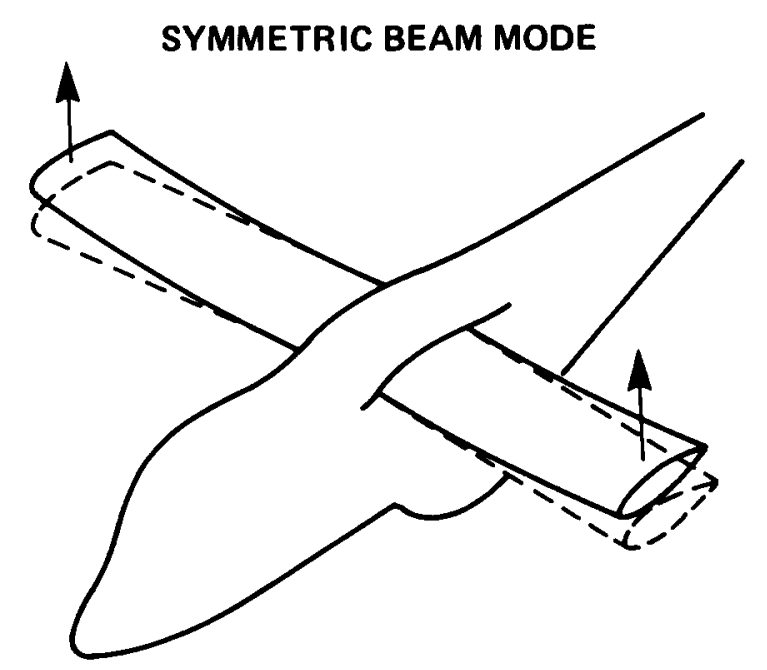

ANTISYMMETRIC BEAM MODE

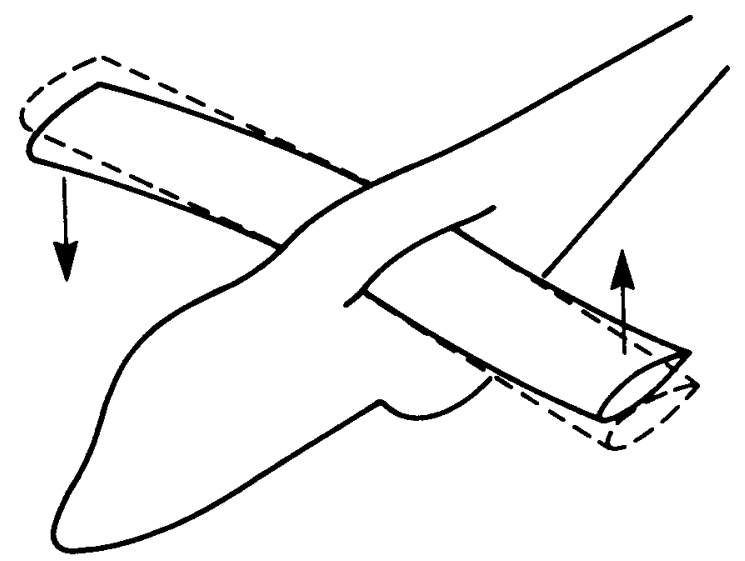

SYMMETRIC CHORD MODE
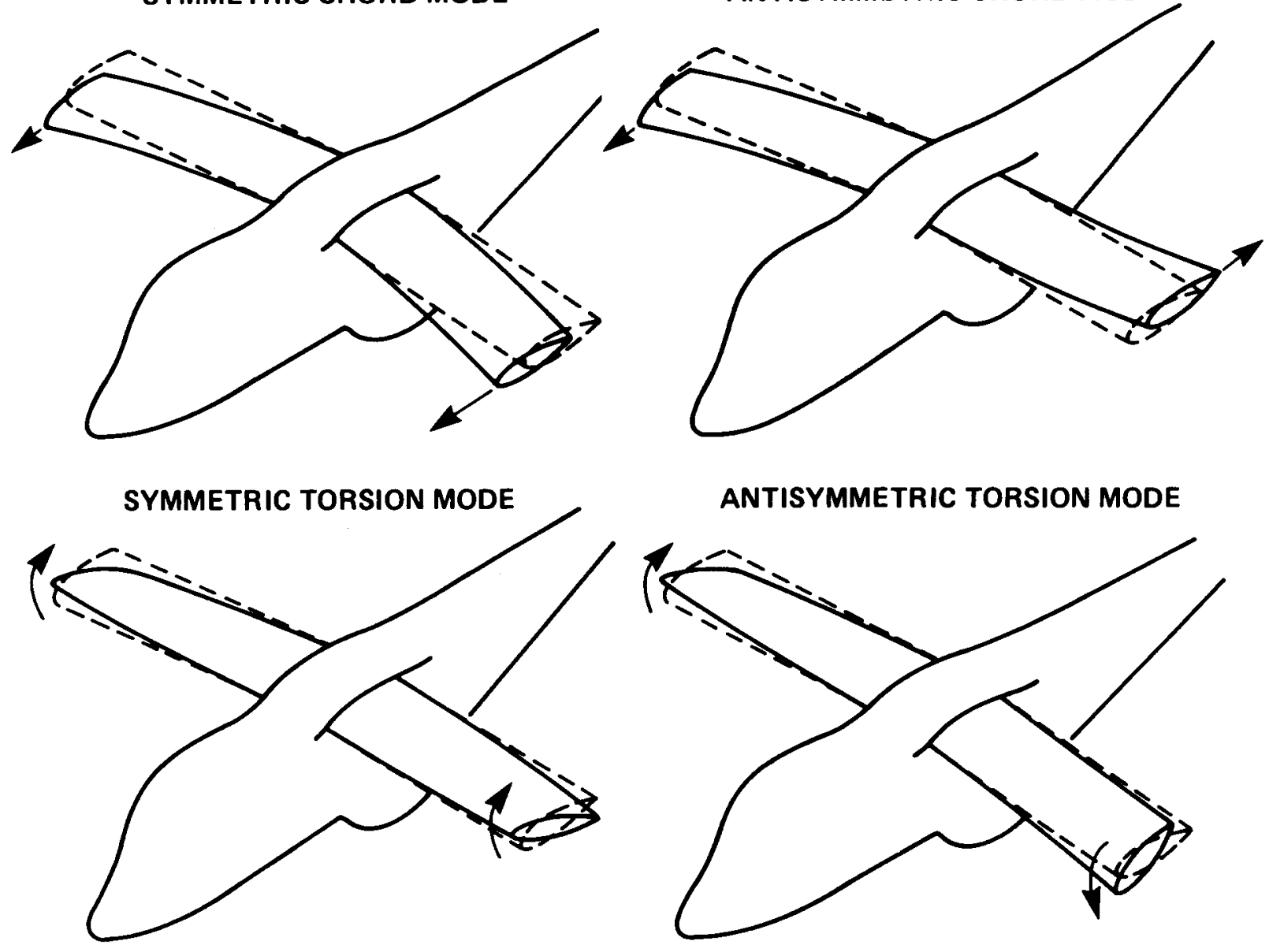

Figure 3.- XV-15 aeroelastic wing modes. 


\section{O PREDICTED FREQUENCY (NASTRAN) \\ $\mapsto$ MEASURED FREQUENCY (NAVFIT)}

WING MODE:

SYMMETRIC BEAM

ANTISYMMETRIC BEAM

SYMMETRIC CHORD

ANTISYMMETRIC CHORD

SYMMETRIC TORSION

ANTISYMMETRIC TORSION

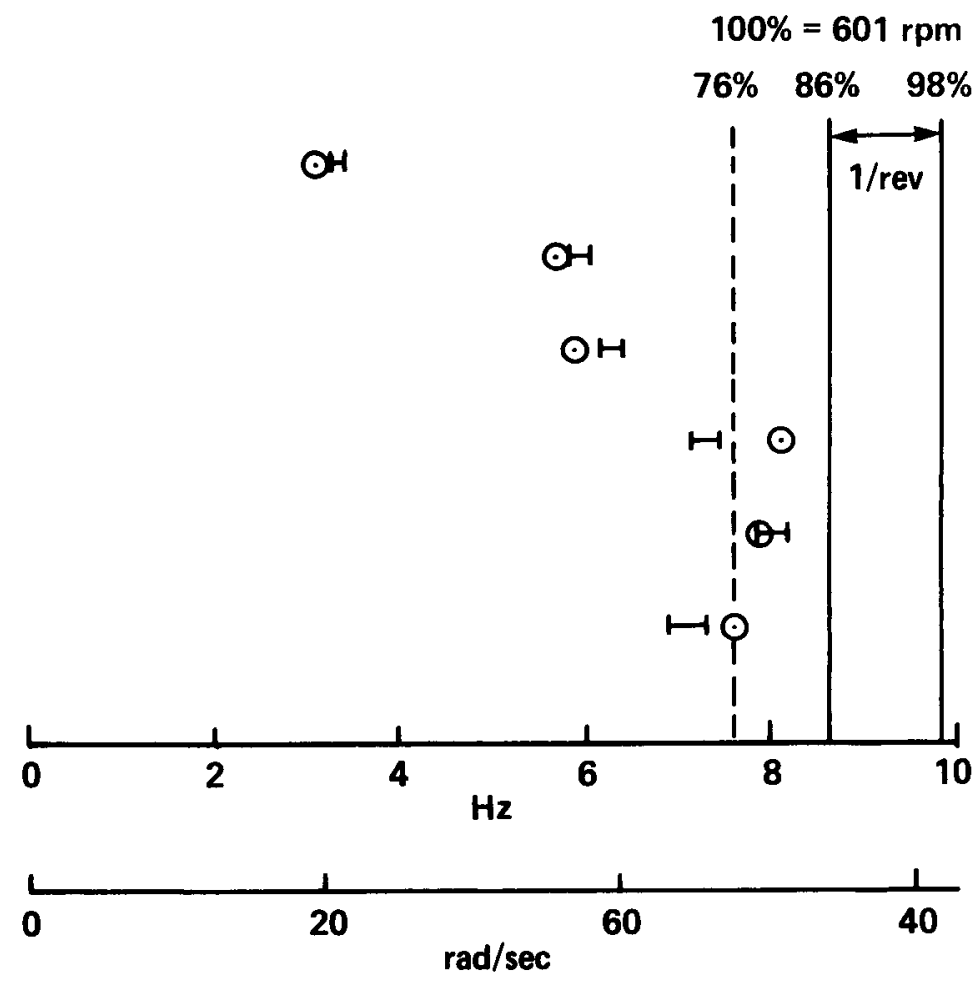

Figure 4.- Ranges of predicted and measured wing frequencies.

torsion modes, and a similar actuator in series with the right-rotor collective control to excite the chord modes. By adding a flaperon exciter to the left wing (fig. 2), different modes could be separately excited more easily. Symmetric modes were excited by driving the flaperons in phase; antisymmetric modes were excited by driving the flaperons in opposite phase. Furthermore, the paired flaperon exciters adequately excited the chord modes without using the collective exciters, which considerably simplified the flight tests. An amplitude of $100 \%$ equalled $\pm 5^{\circ}$ of flaperon motion.

An electronic controller automatically swept the exciters from 1 to $10 \mathrm{~Hz}$, using a logarithmically increasing sweep rate of approximately ten cycles per octave. This was faster than the rate recommended in reference 9, but still slow enough to reveal each mode. Three such sweeps in succession were performed at each test condition.

The flight conditions tested are discussed later in this report under Flight Test Results. 


\section{ANALYSIS METHODS}

The overall concept of the modal identification methods used in this study is to first estimate the frequency response $H(f)$ between the aircraft excitation and structural response, and then to determine the modal damping and frequency by second-order model fitting. Two approaches for obtaining the frequency-response estimates are available; the choice of method depends upon the relative levels of measurable excitation (flaperon input) and unmeasurable excitation (turbulence), as discussed in reference 10 .

Figure 5 illustrates the excitation of the aircraft by measurable and unmeasurable inputs $x(t)$ and $m(t)$; the measured response $y(t)$ is corrupted by measurement noise $n(t)$. If the measurable and unmeasurable inputs and measurement noise are fully uncorrelated, then the unbiased (true average value) frequency response $H(f)$ may be estimated from the cross- and auto-spectral functions $G_{x y}(f)$ and $G_{x x}(f)$ as

$$
H=\frac{G_{x y}}{G_{x x}}
$$

(dependence on frequency $f$ is implied). The random scatter in the estimate is a function of the noise-to-signal ratios $G_{m m} / G_{x x}$ and $G_{n n} / G_{v v}$ (see ref. 10). Thus, when sizeable, persistent, and measurable excitation of a mode is achieved (i.e., small noise-to-signal ratios), the calculation method of equation (1) is preferable; this (direct) approach is referred to herein as the "transfer-function method."

When adequate direct excitation cannot be achieved, as in the case of chordmode response to flaperon inputs, an alternate method is used (fig. 6). In this (indirect) approach, referred to herein as the "cross-spectral method," structural responses from the right and left wings $\left(y_{R}\right.$ and $y_{L}$ ) are cross-correlated with each other, rather than with the flaperon inputs. The resulting cross-spectrum is

$$
G_{y_{R} y_{L}}=H_{R}^{*} H_{L}\left[G_{x x}+G_{m m}\right]
$$

For symmetric modes, $H_{R}=H_{L}$, and for antisymmetric modes, $H_{R}=-H_{L}$. In the symmetric case,

$$
G_{y_{R} y_{L}}=|H|^{2}\left[G_{x x}+G_{m m}\right]
$$

which allows an estimate of the frequency-response magnitude $|H|$ (to within a scale factor) when the total excitation $\left[G_{x x}+G_{m m}\right]$ is constant within the frequency range of the modal response (which is generally a satisfactory assumption). In the crossspectral method, the phase information in $H$ is lost; however, if the noise-tosignal ratio $G_{m m} / G_{x x}$ is large, a much lower scatter in the magnitude estimate is achieved than would be possible with the transfer-function method. The damping 


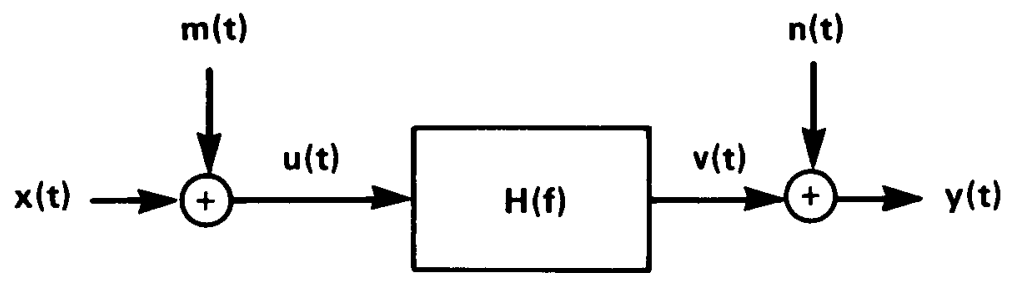

Figure 5.- Signals and noise affecting transfer-function calculations.

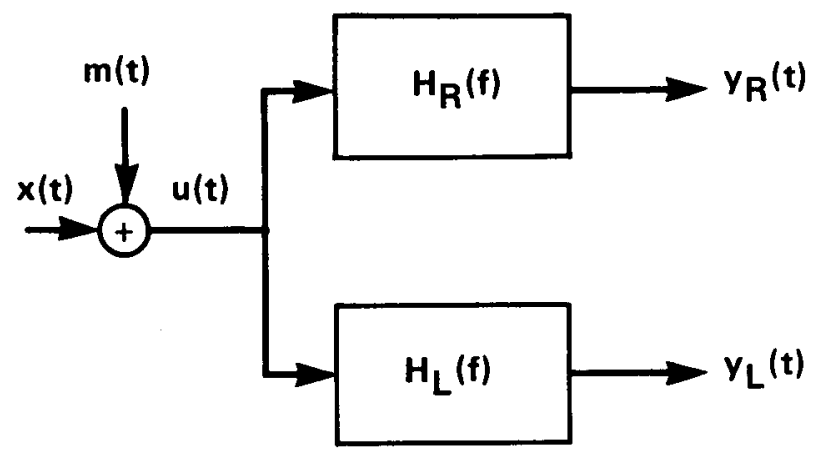

Figure 6.- Signals and noise affecting cross-spectrum calculations.

ratio and frequency are then determined from the magnitude response estimate (demonstrated in ref. 5).

Figure 7 schematically shows the procedures used to conduct the analyses discussed above. Each large block corresponds to a separate computer program. After each flight, the data are loaded into the Tilt-Rotor Engineering Database System (TRENDS) for ease of subsequent access. Next, the Frequency Response Identification (FRESPID) program generates the spectral functions from the time histories in TRENDS. Finally, the modal parameters are determined by using the curve-fitting program, NAVFIT. All computations are performed off-line (postflight).

TRENDS was developed by M. J. Bondi of NASA Ames Research Center and W. S. Bjorkman of Analytical Mechanics Associates, Inc. FRESPID was written by $M$. B. Tischler and J. G. M. Leung of Ames Research Center, and NAVFIT was originally developed by J. Hodgkinson and J. Buckley of McDonnell Douglas Aircraft (ref. 11). For a detailed discussion of FRESPID and NAVFIT, see reference 12 .

The following sections briefly summarize the programs and discuss their application to XV-15 aeroelastics data.

\section{Fourier-Transform Computations}

The first step is to Fourier-transform the exciter and structural response data using FRESPID. For the transfer-function method, corresponding left and right transducers (strain gages or LVDTs) are summed or differenced, depending on the mode, to form composite inputs and outputs. If the two transducers are properly chosen, then the structural signals will be highly correlated and in phase for 


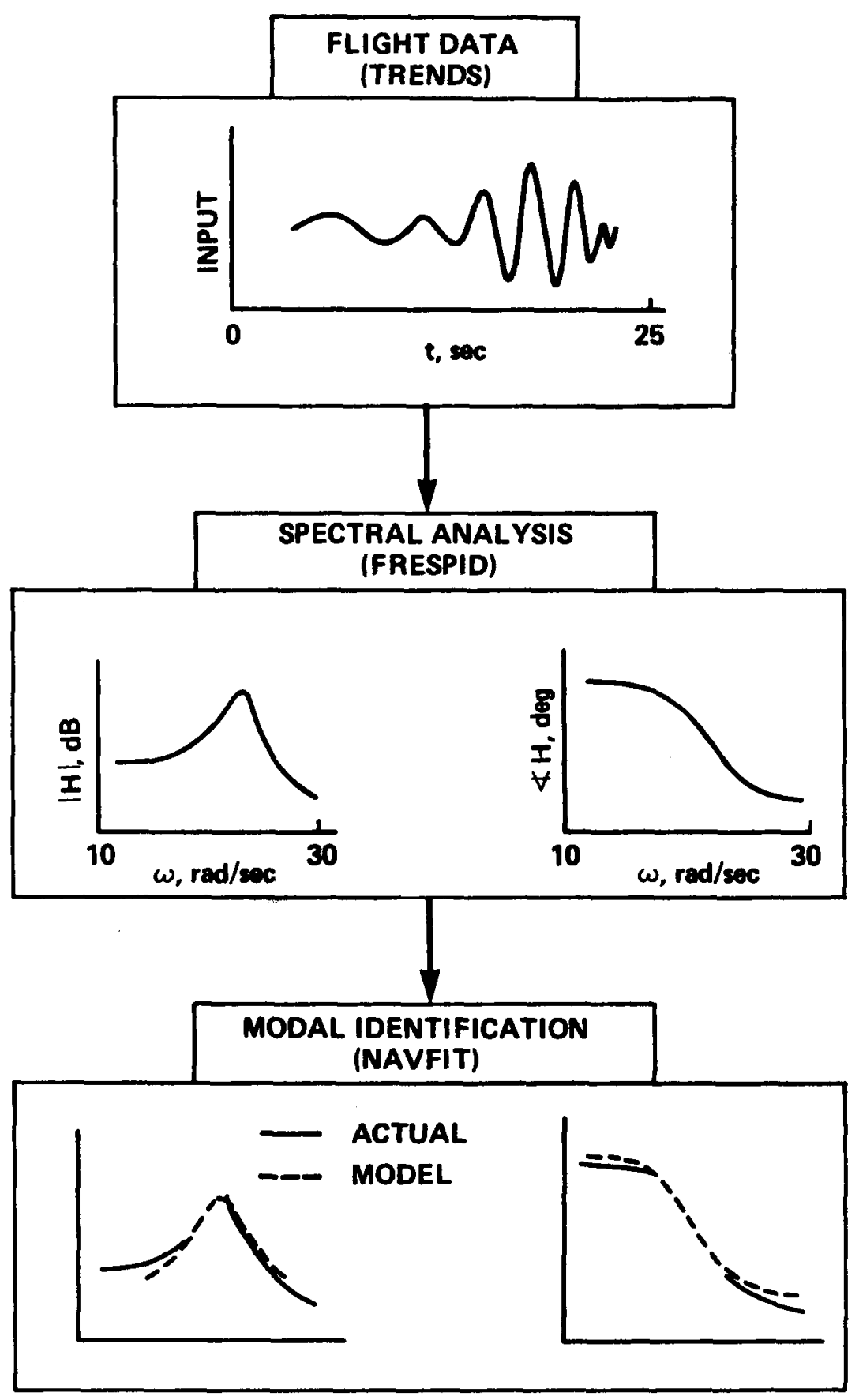

Figure 7.- Sequence of data processing for frequency-domain modal identification.

symmetric modes, and highly correlated but out of phase for antisymmetric modes. Noise will not be correlated; thus corruption of the spectral data is minimized.

FRESPID transforms the time-history data to the frequency domain by using a chirp z-transform. The chirp z-transform algorithm is an improvement on the 
conventional Cooley-Tukey Fast Fourier Transform (FFT), in that it allows arbitrary resolution over a specified frequency range (ref. 13).

Figure 8 shows the computational procedures which are used by FRESPID to generate the auto- and cross-spectral functions from the input and output timehistories. The dc components and linear drifts are first removed to prevent oscillation in the spectral calculations. Multiple runs are concatenated to form composite time-histories. The (concatenated) time-histories are digitally filtered and partitioned into several overlapping sections. Each section is scaled with a cosine weighting function ("Hanning window") to prevent side lobes and leakage (ref. 14). The spectral content of each section is analyzed using the chirp z-transform. The total spectrum is finally determined by averaging the spectra of all of the sections.

\section{Spectral Functions}

Once the Fourier coefficients have been computed by the chirp z-transform, the auto- and cross-spectral functions, $G_{x x}(f), G_{y y}(f)$, and $G_{x y}(f)$, and the transfer function, $\mathrm{H}(\mathrm{f})$, are calculated by the formulas in reference 10 . The coherence function $\gamma^{2}$ is also computed; it is useful for evaluating the identification accuracy (ref. 10). The magnitudes of the spectral functions are converted to power decibels (e.g., $10 \log _{10}\left|G_{x x}(f)\right|$ ) for plotting. The transfer-function results are presented in standard Bode form, that is, a semilog plot of magnitude $\left(d B=20 \log _{10}|H(f)|\right)$ and phase $(\mathrm{deg})$ versus frequency ( $\left.\mathrm{rad} / \mathrm{sec}\right)$.

Figure 9 shows the output time-history of the left wing-bending strain gage produced by a symmetric flaperon sweep (the right signal is nearly identical). The autospectrum $G_{\mathrm{yx}}$ corresponding to three such sweeps is fairly flat, as shown in figure 10. The associated cross-spectrum $G_{y_{R}} y_{L}$ between the right and left signals is plotted in figure 11, which clearly shows the peak of the first bending mode.

Figure 12 shows the transfer-function frequency and phase for the symmetric beam response to flaperon input. The shape of the magnitude peak of the mode is similar, but not identical, to that of the cross-spectrum (fig. 11), because the autospectrum is not precisely constant (fig. 10). The phase plot clearly shows the $90^{\circ}$ change in phase at the natural frequency.

For the transfer-function method, the coherence function $\gamma_{x y}^{2}$ may be interpreted as that fraction of the output (response) spectrum that is linearly related to the input (excitation) spectrum (ref. 14). If the system is perfectly linear and noise-free, the coherence will be unity. For the cross-spectral method, high coherence $\gamma_{y_{R}}^{2}$ implies a common excitation source to the right and left wings, and low output sensor noise. For both methods, the coherence is a good measure of the quality of the data prior to application of the modal curve fit.

Figure 13 illustrates the coherence functions corresponding to the crossspectrum and transfer function shown in figures 11 and 12 . The coherence associated with the cross spectrum is very nearly unity throughout the frequency range of the 


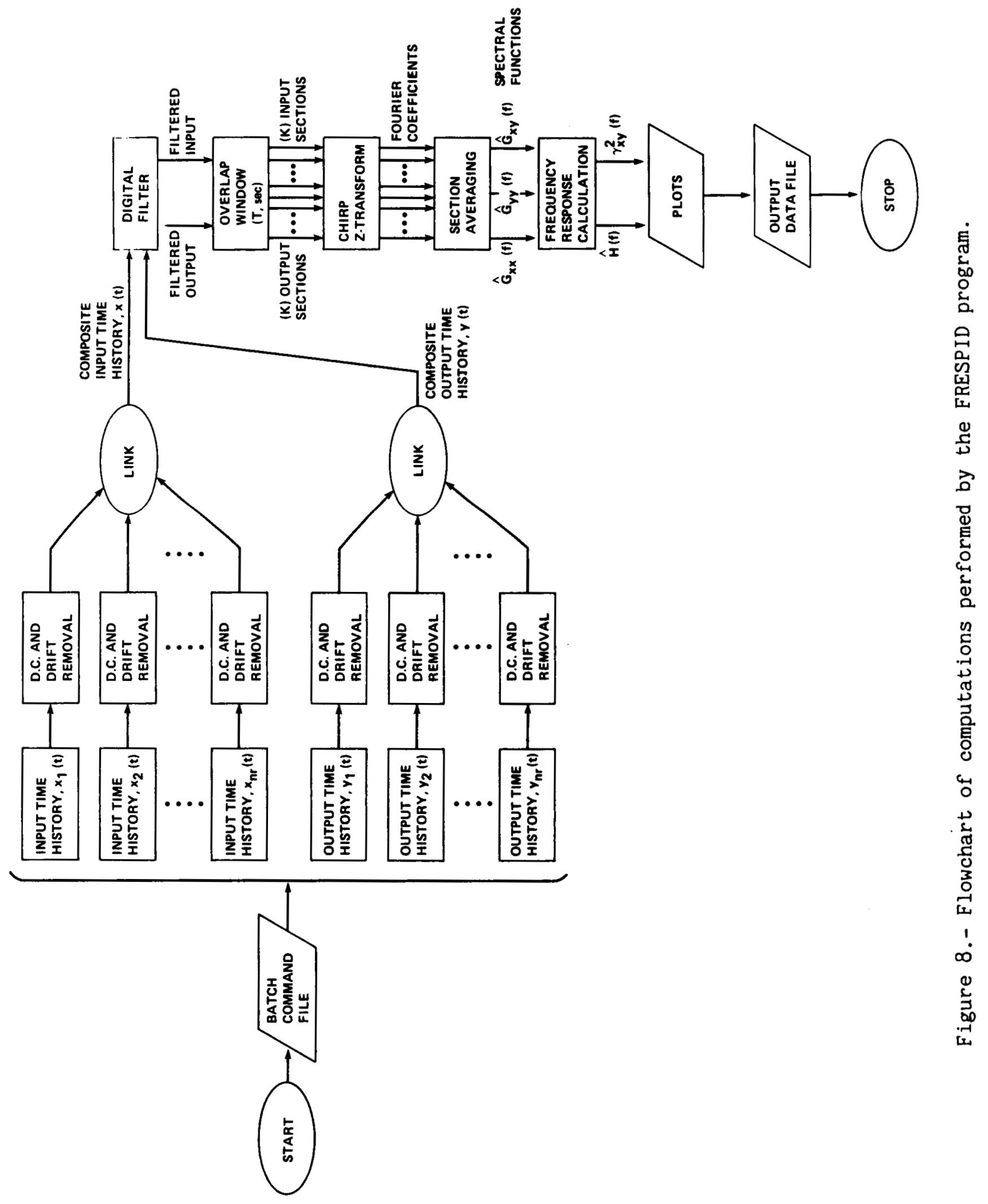




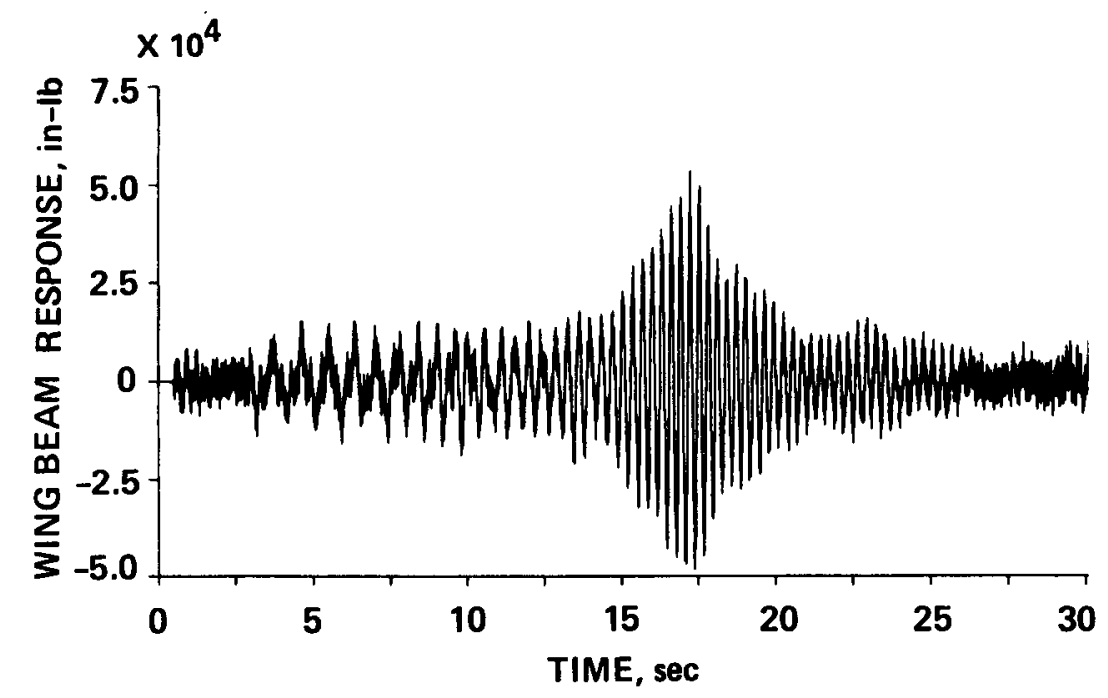

Figure 9.- Strain-gage output for one symmetric flaperon sweep (right-wing beam bending).

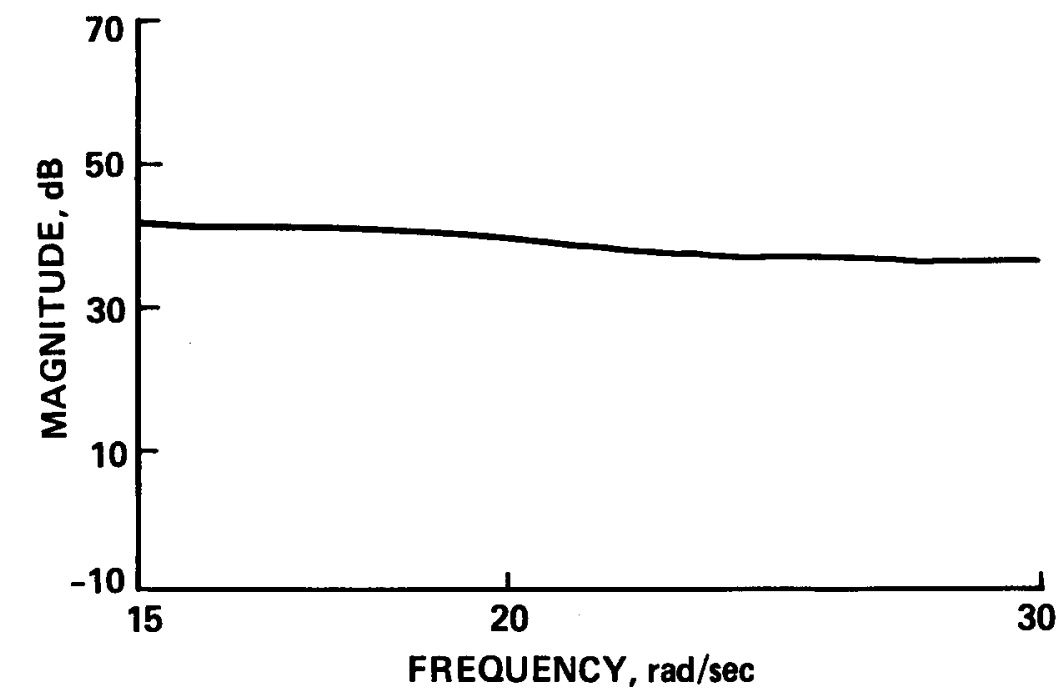

Figure 10.- Autospectrum $G_{x x}$ of the summed flaperon inputs for three symmetric sweeps.

mode, indicating a highly linear response with little noise. However, the values for the transfer-function data are not as good as those for the cross-spectral data, which is a typical result. Worse coherence was often seen in other modes, especially the chord mode, where the response was excited less directly.

\section{Frequency and Damping Calculations}

Once cross-spectra and transfer functions have been calculated by the Fouriertransform program FRESPID, modal frequencies and damping are determined by curvefitting the spectral data. An integration method was also tried for comparison. 


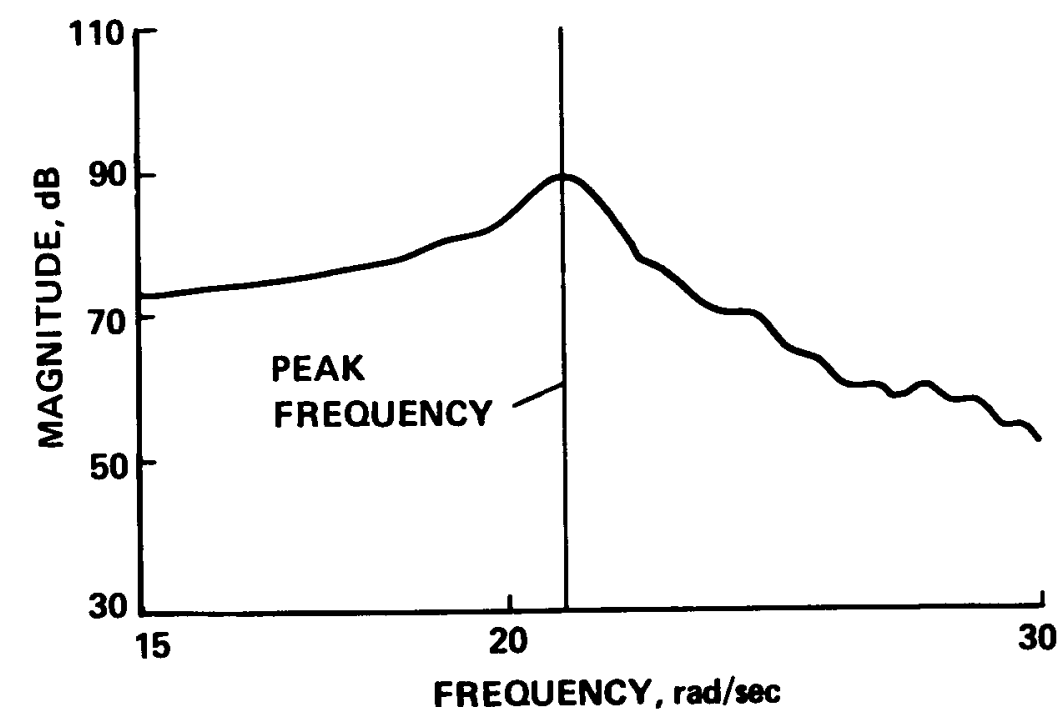

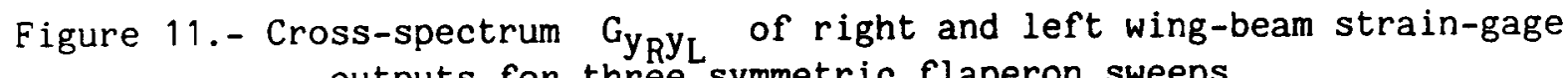
outputs for three symmetric flaperon sweeps.

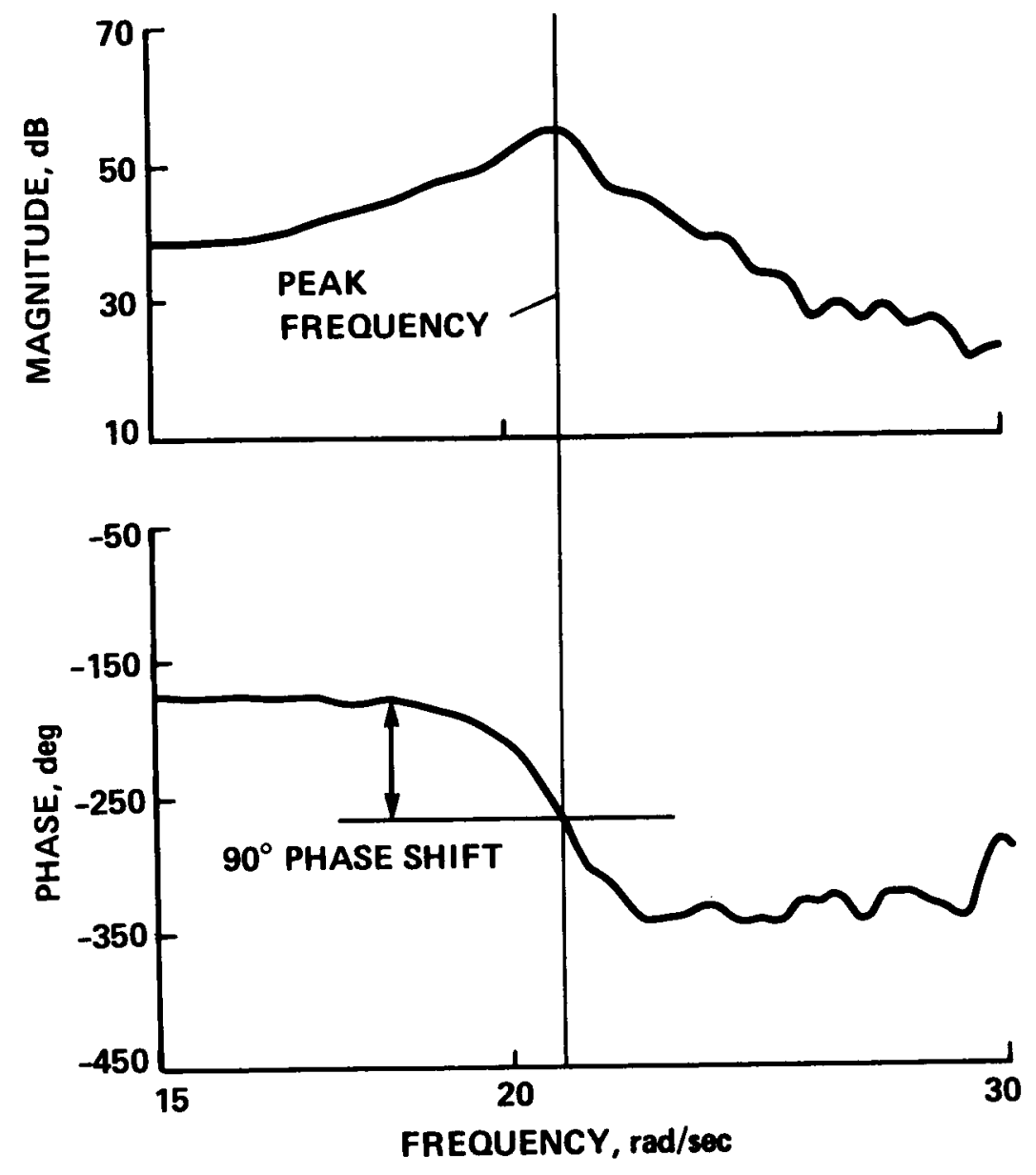

Figure 12.- Transfer function H (magnitude and phase) for the symmetric beam mode. 

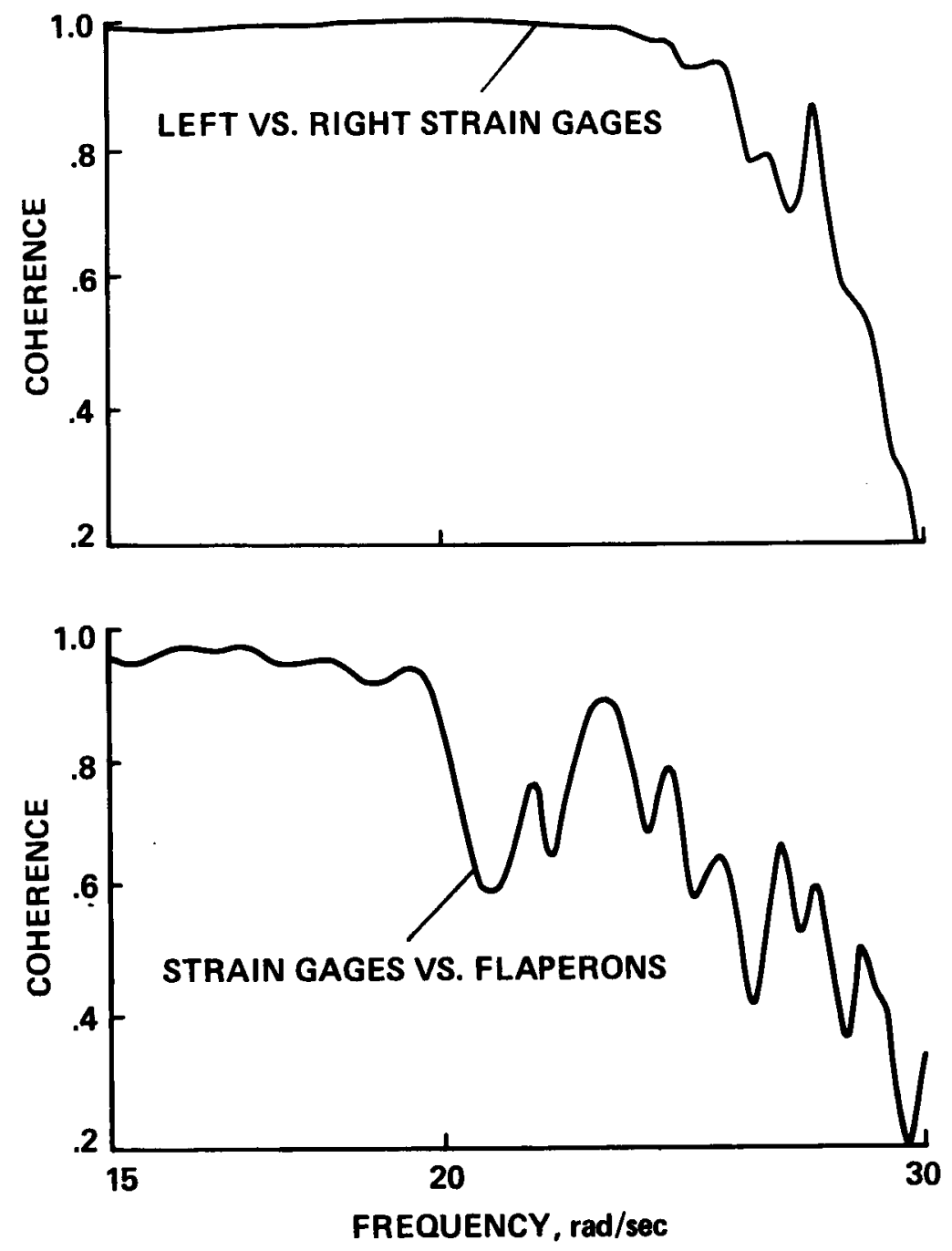

Figure 13.- Coherence functions $r_{y_{R} y_{L}}^{2}$ and $r_{x y}^{2}$ associated with the dross-spectrum ( $\mathrm{fig} .11$ ) and the transfer function ( $\mathrm{fig} .12$ ).

The individual modes are well approximated by second-order quadratic models, which are functions of modal damping $\zeta$ and frequency $\omega_{n}$. Only such models were used in the present study.

The curve-fitting program NAVFIT is a general multimode, high-order analysis using both magnitude and phase data. Either cross-spectral or transfer-function data can be analyzed with NAVFIT as desired. The user specifies a frequency range, the order of the model to be fitted, and (optionally) initial estimates of $\zeta$ and $\omega_{n}$. Phase shifts caused by either unmodeled higher modes or $1 / \mathrm{rev}$ can be fitted with a time delay. An iterative algorithm is used to refine the model by systematically varying its coefficients. As discussed earlier, modal fitting is based on the magnitude and phase for the transfer-function method, but on magnitude alone for the cross-spectral method. 
The model is fitted by minimizing a cost function based on the squares of both magnitude and phase errors. Frequency weighting is used to emphasize the data that are closest to the peak structural response. The relative weights of the magnitude and phase errors are chosen to yield results equivalent to equal weighting of the real and imaginary parts of the complex transfer function (ref. 12). For crossspectral data, the phase weight is set to zero.

In addition to NAVEIT, a method was tried which computed the damping ratio from spectral data by integrating over the spectral peak. The integration program implemented the second-order system analysis discussed in reference 15 for cross-spectra and transfer functions. The algorithm was extended to allow two parameters, $\omega_{n}$ and the peak spectral magnitude, to be iteratively varied to obtain a best fit to the data.

There were thus four possible calculations of frequency and damping: integration and curve fits, each applied with the cross-spectral and transfer-function methods. The final choice was based upon the scatter in estimating $\zeta$ and $\omega_{n}$ at the baseline point (defined below under Flight-Test Results). With one exception, the antisymmetric beam mode, NAVFIT gave the lowest scatter; even in the exceptional case, the NAVFIT results showed no statistically significant differences from the integration method.

The transfer-function method gave better results than the cross-spectral method, except for the chord modes. The flaperons cannot directly excite the wing in the chordwise direction as they can in bending and torsion. The resulting ratio of noise (atmospheric turbulence excitation) to measurable input is high, which explains why the cross-spectral method gives better results for chord modes. Various refinements to the transfer-function method are being explored in hopes of improving its noise rejection for the chord modes.

An example of the use of NAVFIT to determine frequency and damping for the symmetric beam mode is given in figure 14 (compare with fig. 12). Note that magnitude and phase are both fitted with a second-order response.

\section{FLIGHT-TEST RESULTS}

Figure 15 shows the portions of the XV-15 flight envelope covered during the aeroelastics flight tests. Because the aircraft had already been cleared to fly the envelope shown, the frequency sweeps were concentrated within a fairly narrow region so as to more rigorously verify the frequency-domain technique. The most comprehensive data were taken at 10,000 - $\mathrm{ft}$ density altitude at $86 \%$ rotor speed $(8.6 \mathrm{~Hz})$. The speed range was 150 KIAS (Knots Indicated Airspeed), the normal speed for conversion to airplane mode, up to $220 \mathrm{KIAS}$, the torque-limited maximum speed for level flight. Only these data are reported here. (Limited data were also taken at 5,000 $\mathrm{ft}$ and $15,000 \mathrm{ft}$ at $86 \%$ rotor speed, and at $10,000 \mathrm{ft}$ at $98 \%$ rotor speed. Maximum-power climbs and power-off descents were performed at 150 KIAS at $86 \%$ rotor speed, with data taken as the aircraft passed 10,000 ft.) 

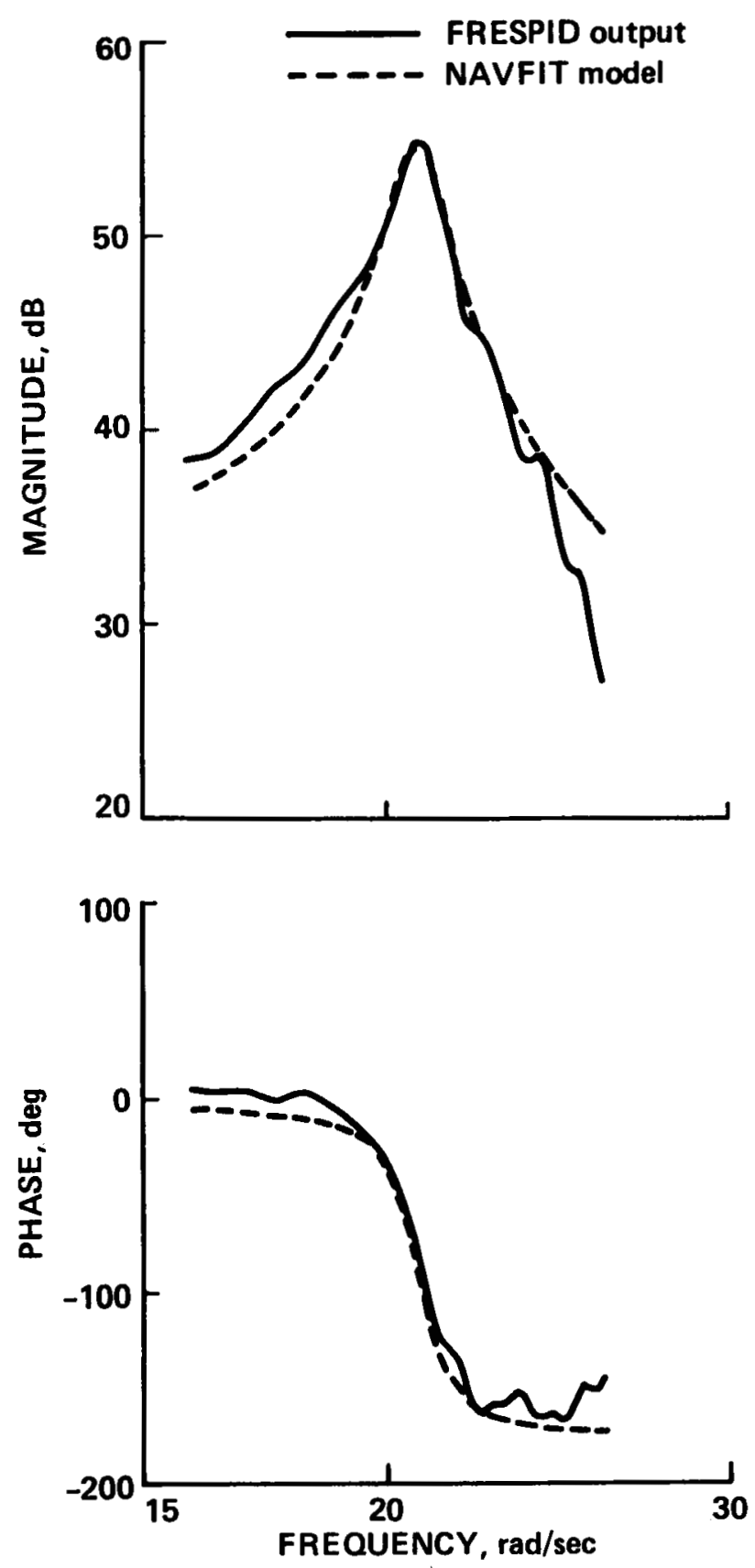

Figure 14.- Curve fits to transfer-function magnitude and phase for the symmetric beam mode.

Limitations on flight-test time did not permit replications of all test points. Therefore, a baseline point of $150 \mathrm{KIAS}$ at $10,000 \mathrm{ft}$ was chosen as an easily repeatable flight condition to explicitly test for scatter in the frequency and damping estimates.

Earlier flight tests (ref. 6) showed interaction of the Stability Control Augmentation System (SCAS) with modal responses, which was eliminated by 


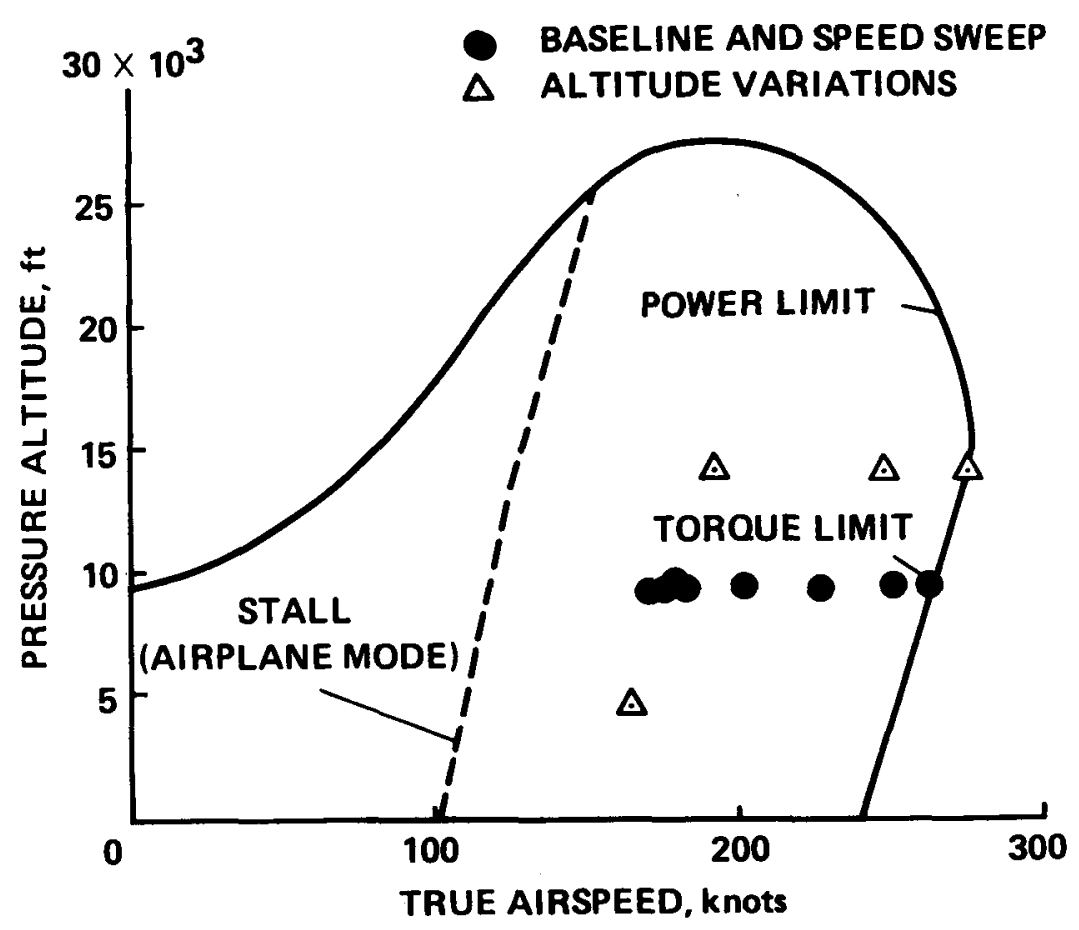

Figure 15.- XV-15 flight envelope, showing frequency-sweep test points.

modification of the SCAS. To ensure that there were no other interactions, each part of the automatic flight control system--the SCAS, the Attitude Retention System (ARS), and the Force Feel System (FFS)--was individually turned off during three series of sweeps at the baseline point. In a comparison of the results with the other baseline estimates, no statistically significant differences were noted. These data were subsequently included in the baseline data.

\section{Summary Plots and Statistics}

Figure 16 summarizes the frequency and damping results for all six modes. Open symbols are estimates made with exponential-decay methods (from ref. 16); closed symbols are frequency-domain estimates. (Reference 16 does not always give an explicit frequency estimate corresponding to each damping estimate.) The frequencydomain method yields low scatter at the baseline point and good consistency between airspeeds, hence higher reliability than the exponential-decay method. (Individual modes are discussed in detail below.)

A detailed assessment of all available predictive methods is beyond the scope of this paper. To illustrate discrepancies between predictions and flight data, representative results of two different analyses are shown in figure 16 . The DYN 4 predictions of frequency and damping were made by Bell Helicopters (ref. 16); the CAMRAD predictions were made by NASA. The CAMRAD model used here is that of reference 3 , but it was modified to have the correct precone, rotor speed, and flight conditions. Neither analysis exactly models the aircraft. However, the trends in 


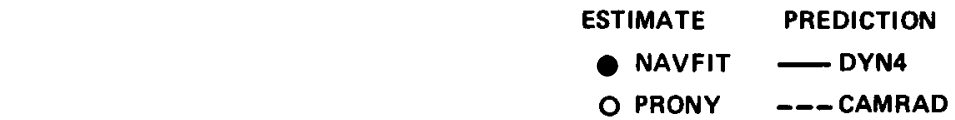

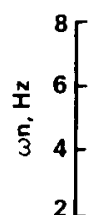

SYMMETRIC WING BEAM MODE
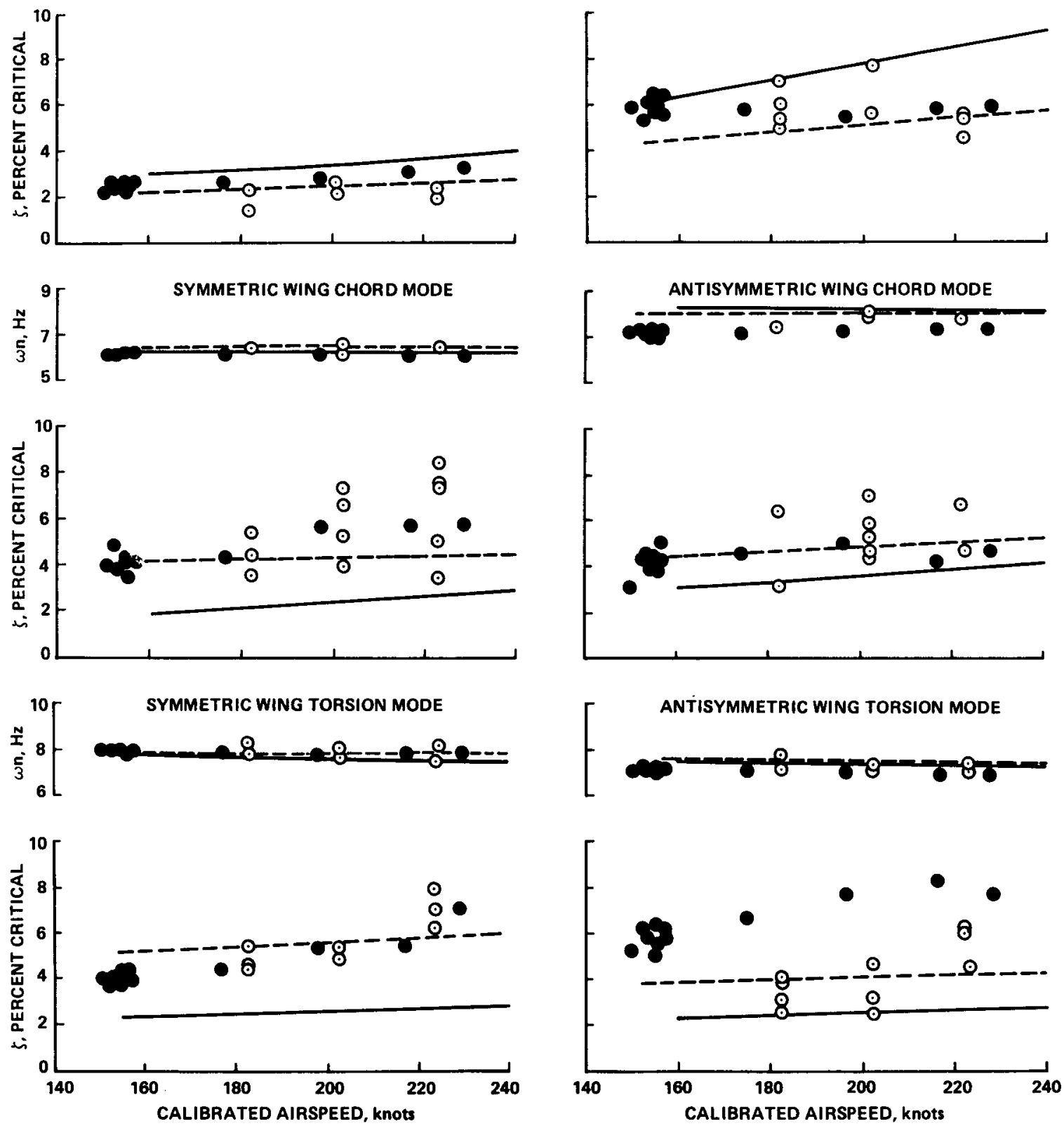

Figure 16.- Summary of aeroelastic wing-mode measurements and predictions. 
the plotted results illustrate the differences between the two methods. Furthermore, the DYN4 program does not represent the latest analytical techniques used by Bell Helicopters, but it is the method used during the design and initial flight test of the $\mathrm{XV}-15$.

Numerical results of the frequency-domain method are summarized in Table 1 for the 150-KIAS baseline point. Listed for each mode are the averages of damping $(\bar{\zeta})$ and frequency $\left(\bar{\omega}_{n}\right)$ and their respective standard deviations $\left(\sigma_{\zeta}\right)$ and $\left(\sigma_{\omega}\right)$, which are measures of the scatter among the estimates. The standard deviations of the damping range from $6 \%$ to $15 \%$ of the average values, while the standard deviations of the frequency are all less than $1 \%$.

TABLE 1.- XV-15 WING MODES AT THE BASELINE FLIGHT CONDITION (STATISTICS ARE BASED ON 8 DATA POINTS)

\begin{tabular}{|c|c|c|c|c|c|c|}
\hline Mode & $\bar{\zeta}$ & $\sigma_{\zeta}$ & $\sigma_{5}$ & $\overline{\omega_{n}}$ & $\sigma_{\omega}$ & $\sigma_{\omega}$ \\
\hline & $\begin{array}{l}\text { critical } \\
\text { damping }\end{array}$ & $\begin{array}{l}\text { f critical } \\
\text { damping }\end{array}$ & \% error & $\mathrm{Hz}$ & $\mathrm{Hz}$ & terror \\
\hline Symmetric Beam & 2.52 & 0.218 & 8.6 & 3.31 & 0.0107 & 0.32 \\
\hline Antisymmetric Beam & 5.97 & 0.401 & 6.7 & 5.89 & 0.0427 & 0.73 \\
\hline Symmetric Chord & 4.17 & 0.398 & 9.5 & 6.28 & 0.0198 & 0.32 \\
\hline Ant isymmetric Chord & 4.06 & 0.609 & 15.0 & 7.21 & 0.0440 & 0.61 \\
\hline Symmetric Torsion & 4.08 & 0.245 & 6.0 & 8.07 & 0.0385 & 0.48 \\
\hline Antisymmetric Torsion & 5.77 & 0.464 & 8.0 & 7.24 & 0.0349 & 0.48 \\
\hline
\end{tabular}

In a very few cases--notably, antisymmetric torsion--a significant fraction of the scatter can be explained by weight changes caused by fuel burnoff. It is not practical to collect all flight data at exactly the same fuel state. Therefore, the values given in table 1 represent realistically achievable performance of the frequency-domain method.

There is no comprehensive set of exponential-decay data corresponding to the frequency-sweep baseline data. In order to make statistical comparisons between the results of the two flight-test methods, the frequency and damping results were curve-fitted against airspeed. This procedure allows consistent comparisons to be made using all of the data. Linear fits were used, partly because all predictions show nearly constant slopes within the airspeed range and partly because the standard error of each fit will be a conservative measure of scatter if the true variations are in fact nonlinear.

The standard errors, slopes, and intercepts of the damping curve fits are listed in table 2 for each mode. The standard error is a measure of the scatter about a fitted curve (analogous to the standard deviation about a point). The frequency-domain method generally has a standard error of one-half or less than the exponential-decay method. 
TABLE 2.- CURVE-FIT STATISTICS OF DAMPING VERSUS AIRSPEED

\begin{tabular}{|c|c|c|c|c|c|c|c|c|}
\hline \multirow[t]{2}{*}{ Mode } & \multicolumn{4}{|c|}{ Erequency-Domain Method } & \multicolumn{4}{|c|}{ Exponential-Decay Method* } \\
\hline & $\begin{array}{l}\text { No. of } \\
\text { Points }\end{array}$ & $\begin{array}{l}\text { Standard } \\
\text { error, \% } \\
\text { critical }\end{array}$ & $\begin{array}{l}\text { Intercept, } \\
\% \text { critical } \\
\text { damping }\end{array}$ & $\begin{array}{l}\text { Slope } \\
q / \text { knot }\end{array}$ & $\begin{array}{l}\text { No. of } \\
\text { points }\end{array}$ & $\begin{array}{l}\text { Standard } \\
\text { error, } \% \\
\text { critical }\end{array}$ & $\begin{array}{l}\text { Intercept, } \\
\text { \% critical } \\
\text { damping }\end{array}$ & $\begin{array}{l}\text { Slope } \\
\text { \%/knot }\end{array}$ \\
\hline Symmetric Beam & 12 & .181 & 1.06 & .00947 & 6 & 0.422 & 0.865 & .0069 \\
\hline Antisymmetric Beam & 12 & .352 & 6.06 & -.000776 & 9 & 1.02 & 8.82 & -.015 \\
\hline Symmetric Chord & 12 & .405 & 0.442 & .0405 & 12 & 1.64 & -4.61 & .050 \\
\hline Antisymmetric Chord & 12 & .565 & 2.84 & .00823 & 9 & 1.64 & 0.883 & .022 \\
\hline Symmetric Torsion & 12 & .332 & -1.39 & .0353 & 8 & 0.491 & -5.36 & .056 \\
\hline Antisymmetric Torsion & 12 & .446 & 0.557 & .0341 & 10 & 1.01 & -5.75 & .049 \\
\hline
\end{tabular}

The key requirement is to detect any change in the trend of damping with airspeed as a stability boundary is approached. Accurate estimates of average damping are not as important as accurate estimates of slope, against which deviations can be detected. Most modes show increasing damping with airspeed, but will change to a negative slope at a sufficiently high airspeed (see refs. 3 and 6). For some modes, the negative slope is predicted to become very steep at the critical airspeed for flutter.

Figure 16 and tables 1 and 2 together show that the frequency-domain method is very reliable and completely adequate to detect a significant decrease in damping should a dangerous part of the flight envelope be entered. Even without further refinement of the flight-test or analytical techniques, the new approach is seen to be preferable to the exponential-decay method.

Care should be taken when comparing the exponential-decay results with the new frequency-sweep results. No complete set of the former type of data for an aircraft configuration exists that exactly matches that for the new data. The exponentialdecay results shown in figure 16 are for $1.5^{\circ}$ precone titanium hubs; the frequencydomain results are for $1.5^{\circ}$ steel hubs. Both configurations are for the aircraft at NASA Ames with metal blades. These are the most closely-matched aeroelastics data sets available. Other reports on XV-15 aeroelastics (e.g., refs. 6, 7, and 16) sometimes include data for the aircraft operated by Bell Helicopters, which is not identical to the aircraft at NASA, or for $2.5^{\circ}$ precone hubs. These additional configurations are thought to have slightly different aeroelastic behavior.

\section{Individual Modes}

For the symmetric beam mode, the new frequency-domain estimates of natural frequency $\omega_{n}$ are similar to the old exponential-decay results, and closely follow the predictions. The new estimates of damping $\zeta$ show a very consistent increase with airspeed. The CAMRAD estimate appears to match the new estimates of $\omega_{n}$ slightly better than DYN4, but the match for $\zeta$ is slightly worse. However, the 
differences between the slopes of DYN4 and CAMRAD are too small to allow a reliable choice of one method over the other.

For the antisymmetric beam mode, the new estimates of $\omega_{n}$ show less scatter than the old estimates and are slightly lower in frequency than the predictions, especially for CAMRAD. The new estimates of $\zeta$ are much better than the old, but neither set of estimates matches the slope of either predictive method. However, CAMRAD does better approximate the measured slope of $\boldsymbol{\zeta}$.

For the symmetric chord mode, the new estimates of $\omega_{n}$ have slightly less scatter than the old, and are slightly lower in frequency than the predictions. The new estimates of $\zeta$ are much improved, but there is still noticeable scatter. There is too much scatter in even the new estimates to reliably favor either predictive method. It is not known whether the leveling off in the new estimates of $\zeta$ is an artifact of the scatter or an accurate measurement of a nonlinear variation with airspeed; more flight data are required to draw a clear conclusion.

For the antisymmetric chord mode, the new estimates of $\omega_{n}$ are slightly better, but some scatter remains. The estimates are about $0.5 \mathrm{~Hz}$ lower than the predictions and show an increase with airspeed not matched by the predictions. The new estimates of $\zeta$ are better, but scatter is noticeable. The slope in the estimates of $\zeta$ is not clear enough to reliably reveal any errors in the predictions.

For the symmetric torsion mode, the new estimates of $\omega_{n}$ are very good. They do not show the predicted decrease in $\omega_{n}$ as airspeed is increased from the baseline condition (this is more noticeable for DYN4 than for CAMRAD). The new estimates of $\zeta$ are slightly better than the old. Both sets of estimates show increasing slope with airspeed, but it has not been proven that this indicates a real aeroelastic phenomenom. Neither DYN4 nor CAMRAD matches the slope of the estimates.

For the antisymmetric torsion mode, the new estimates of $\omega_{n}$ are definitely better. They are lower than the predicted values, but approximately match the slope predicted by CAMRAD. The new estimates of $\zeta$ are better than the old, and have almost twice the magnitude. Both predictions of $\zeta$ show lower slopes than the estimates. The new estimates show a decrease in slope at the last, highest-speed point, whereas the old estimates appear to show an increase in slope. It cannot be determined whether either case reflects a true change in slope or whether both are merely caused by scatter.

Overall, there is no obvious, consistent pattern of correspondence between either predictive method and the modal estimates based on flight data. The occasional large inconsistencies, known modeling errors (thought to be small), and newly available, improved analyses have prompted a rework of the aeroelastic predictions, which is now in progress. In any event, the excellent results of the frequencydomain analyses should allow reliable evaluations of new predictions. 


\section{CONCLUSIONS}

Frequency-domain estimates of modal frequencies and damping were consistent with airspeed and were highly repeatable at a reference flight condition (within less than $1 \%$ for $\omega_{n}$ and $15 \%$ for $\zeta$ ). These results demonstrate that the frequency-sweep method is a reliable and efficient way of determining XV-15 aeroelastic behavior from flight data. The ability to use either cross-spectral or transfer-function data provides great analytical flexibility, permitting good estimations of all modes. Such analytical methods can now be routinely used to support planned flight tests of the new XV-15 composite blades (ATBS) or any other new configuration.

\section{REFERENCES}

1. Hall, W. E.: Prop-Rotor Stability at High Advance Ratios. Journal of the American Helicopter Society, vol. 11, no. 2, pp. 11-26, April 1966.

2. Edenborough, H. K.: Investigation of Tilt Rotor VTOL Aircraft Rotor-Pylon Stability. AIAA Paper 67-17, presented at the 5th Aerospace Sciences Meeting, New York, N.Y., Jan. 23-26, 1967; and Journal of Aircraft 5(6), MarchApril 1968.

3. Johnson, W.; Lau, B. H.; and Bowles, J. V.: Calculated Performance, Stability, and Maneuverability of High-Speed Tilting-Prop-Rotor Aircraft. NASA TM-8834), Sept. 1986.

4. Alexander, H. R.; Maisel, M. D.; and Giulianetti, D. J.: The Development of Advanced Technology Blades for Tiltrotor Aircraft. Presented at the $11 \mathrm{th}$ European Rotorcraft Forum, London, England, Sept. 10-13, 1985.

5. Tischler, M. B.; Leung, J. G. M.; and Dugan, D. C.: Frequency-Domain Identification of XV-15 Tilt-Rotor Aircraft Dynamics. Presented at the 2nd AIAA/AHS/IES/SETP/SFTE/DGLR Flight Testing Conference, Las Vegas, Nev., Nov. $16-18,1983$.

6. Schroers, L. G.: Dynamic Structural Aeroelastic Stability Testing of the XV-15 Tilt Rotor Research Aircraft. NASA TM-84293, Dec. 1982.

7. Bilger, J. M.; Marr, R. L.; and Zahedi, A.: In-Flight Structural Dynamic Characteristics of the XV-15 Tilt Rotor Research Aircraft. AIAA Paper 81-0612.

8. Representation and Analysis of Sonar Signals. Volume 1: Improvements in the Complex Exponential Signal Analysis Computational Algorithm. Texas Instruments, Inc. Report No. U1-829401-5, June 1971. 
9. Flannelly, W. G.; Fabunmi, J. A.; and Nagy, E. J.: Analytical Testing. NASA CR-3429, May 1981.

10. Bendat, J. S.; and Piersol, A. G.: Engineering Applications of Correlation and Spectral Analysis. John Wiley and Sons, Inc., New York, N.Y., 1980.

11. Hodgkinson, J.; and Buckley, J.: NAVFIT General Purpose Frequency Response Curve Fit (Arbitrary Order). McDonnell Aircraft Company, St. Louis, Mo., Oct. 1978.

12. Tischler, M. B.: Frequency-Response Identification of XV-15 Tilt-Rotor Aircraft Dynamics. NASA TM 89428, May 1987; and USAAVSCOM TM-87-A-2.

13. Rabiner, L. R.; and Gold, B.: Theory and Application of Digital Signal Processing. Prentice-Hall, Inc., Englewood Cliffs, N.J., 1975.

14. Otnes, R. K.; and Enochson, L.: Applied Time Series Analysis. John Wiley and Sons, Inc., New York, N.Y., 1978.

15. Johnson, W.: Development of A Transfer Function Method for Dynamic Stability Measurement. NASA TN D-8522, July 1977.

16. Arrington, W. L.; Kumpel, M.; Marr, R. L.; and McEntire, K. G.: XV-15 Tilt Rotor Research Aircraft Flight Test Data Report. NASA CR 177406, June 1985; and USAAVSCOM TR-86-A-1. 


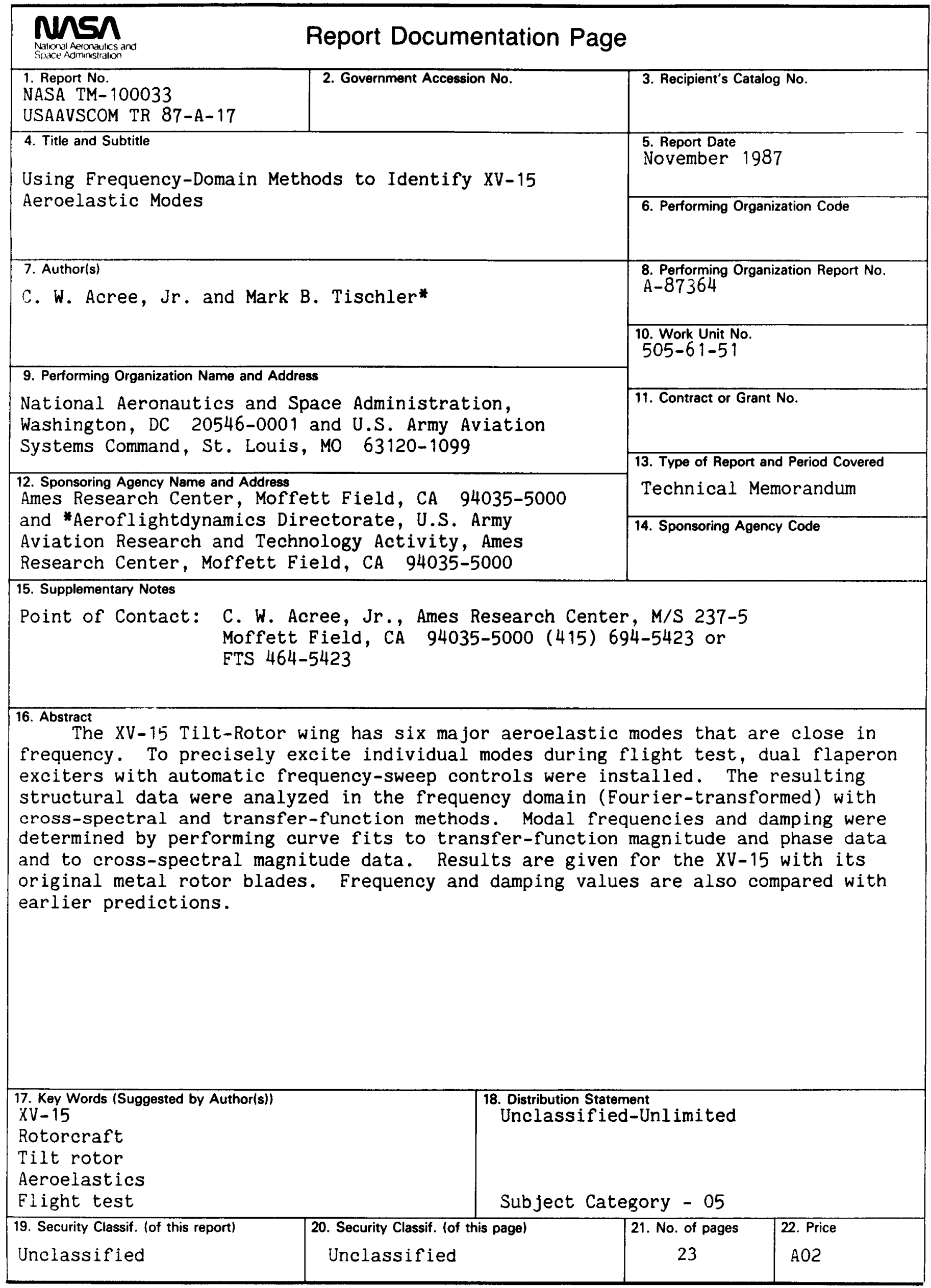

